\title{
Minimizing lost-work costs in a mixed-model assembly line
}

\author{
Bautista $\mathbf{J}^{1}$, Alfaro-Pozo $\mathbf{R}^{1}$, Batalla-García $\mathbf{C}^{1}$
}

\begin{abstract}
Mixed-model assembly lines present two issues due to differences in processing times from product types; these issues are the work overload or unfinished work and the useless time or unproductive time. Within this context, we present, in this paper, a new mathematical model for the mixed-model sequencing problem. This model minimizes the costs by lost production and idle productive time. The model also allows processors carry out their workload with a factor activity greater than the normal, in order to reduce the work overload if it is necessary. Obviously it is also considered to provide economic compensation to workers based on their level of activation. Finally, the model is evaluated by a computational experience linked to a real case from the automotive industry.
\end{abstract}

Keywords: Assembly line; Sequencing; Work overload; Useless time; Costs.

\section{Introduction}

Currently there are many productive systems with Mixed-Model Assembly Lines (MMALs). We find some examples in the automotive industry, or door-lock industry, among others (Bautista, Cano and Alfaro, 2012; Lin and Chu, 2014).

These production systems are characterized by high flexibility, because they are able to assemble different product types. This variety in the product portfolio means that both the consumption of components and the use of resources may differ from one type of product to another. The latter can cause, on the one hand, the existence of unbalanced stock levels throughout the working day; and, on the

\footnotetext{
${ }^{1}$ Joaquín Bautista Valhondo; Rocío Alfaro Pozo; Cristina Batalla García (e-mail: joaquin.bautista@upc.edu;rocio.alfaro@upc.edu; cristina.batalla@upc.edu) Dpto. de Organización de Empresas. Escuela Técnica Superior de Ingeniería Industrial de Barcelona. Universitat Politècnica de Catalunya·BarcelonaTech. Avda. Diagonal, 647. Barcelona. This work was funded by the Ministerio de Economía y Competitividad (Spanish Government) through the FHI-SELM2 (TIN2014-57497-P) project.
} 
other hand, the non-uniform distribution of the workload throughout the working day at workstations.

For these reasons the Mixed-Model Sequencing Problem (MMSP) is especially important in MMALs; while it is true that almost any sequence of product mix is technically feasible, not all sequences have the same economic impact because the component consumptions and the load distribution will be one or another depending on the sequence (Boysen, Fliedner and Scholl, 2009).

A sequence with a non-uniform distribution of workloads may involve both the appearance of work overload, $W$, as the occurrence of useless time, $U$; consecutive sequencing of product units with processing times longer than the time that has the workstation to work on a unit (cycle time, $c$, measured at normal activity, $\alpha^{N}=1.0$ ) can cause the processor does not finish the required work and thus it generates work overload, even though stations have more time to retain the product unit (i.e., time window $l_{k}-c \geq 0$ ); conversely, if processing times are less than the cycle time, the processor will finish operation on the product unit before cycle time completion and therefore it generates useless time.

This paper is adressed to solve the MMSP avoiding simultaneously the work overload and the useless time. To this purpose, it is presented a new mathematical model whose objective is the minimization of the costs generated by unfinished work and time not used by processor to work on any product unit.

In addition, considering that processors are human resources, we can state that their performance varies throughout the workday. Therefore it is possible to consider that processing times are variable in regard with the work pace or activity of operators into the proposed model, such as it is considered in Bautista, Alfaro, Batalla et al. (2014), Bautista, Alfaro, Batalla (2015a) and Alfaro-Pozo (2015). In this way the completed work, $V$, will be increased and the unfinished work, $W$, will be reduced, favoring the original equivalent objectives from the MMSP-W (Yano and Rachamadugu, 1991; Scholl, Klein and Domschke, 1998). Obviously, the gains obtained by reduction of work overload and useless time will be used to compensate the activation of workers.

Finally, a case study linked with the Nissan's Engine Plant in Barcelona is used to evaluate the proposed mathematical model. Specifically, from a demand plan that corresponds to a workday, the gains in work overload and useless time will be compared with the reference models.

\section{The MMSP. Reference Models}

The MMSP constists of establising a bijection between the elements of a $\mathrm{T}$ set (that we enumerate $t=1, \ldots, T$ ) of production cycles and the elements of a $\Psi$ set of products. The elements of $\Psi$ can be grouped in exclusive classes that fulfill 
$\Psi=\cup_{i \in I} \psi_{i}$ and $\psi_{i} \cap \psi_{i^{\prime}}=\{\varnothing\} \quad \forall\left(i, i^{\prime}\right) \in I$, where $I$ is the set of product types (that we enumerate $i=1, \ldots,|I|$ ).

This assignment, of products types to production cycles, is subject to some optimization criterion. There are many criteria, such as the minimization of utility work or inefficiency costs (i.e., idle time, concentration of high workloads)(Fattahi and Salehi, 2009), among others. Indeed, many researches simultaneously optimize more than one criterion or include additional conditions, such as Bautista, Alfaro-Pozo and Batalla-Garcia (2105b). In that research and others (Bautista, Alfaro, Batalla et al. 2014; Bautista, Alfaro-Pozo and Batalla-Garcia, 2105a), the authors have extended the models for the MMSP by considering human factors. Specifically, the authors proposed the $M 4 \cup 3_{-} \dot{\alpha} I$ model to minimize the work overload by means of processors' activation, according to a set of functions for the work pace and the collective bargaining agreements. This model is an extension from the $M 4 \cup 3$ model without activity factors.

Similar to the $M 4 \cup 3 \_\dot{\alpha} I$ model, we consider, variable-processing times in regard with the activity factor; but unlike the reference model, $M 4 \cup 3_{-} \dot{\alpha} I$, in this paper, the processors' activation does not be prefixed. Now, the activity is only limited by the maximum and minimum allowable values. In this way, each processor works with an activity factor depending on the workload at each moment. Therefore, the useless time is not affected by the demanded activation, and both issues, work overload and useless time, are simultaneously minimized.

\section{Minimizing the unproductive costs maximizing productivity}

From the $M 4 \cup 3 \_\dot{\alpha} I$ model (Bautista, Alfaro-Pozo and Batalla-Garcia, 2015a), we propose a model that minimizes the costs of work overload and useless time. The new model is able to activate processors in order to minimize the work overload but this activation must be between the minimum and maximum values allowable for the activity factor. The free activation will lead to unsynchronized workstations regarding the work pace.

The new parameters and variables used in the new model, which is proposed in this work, are the following:

Parameters

\footnotetext{
$L_{k}$ Physical time of presence of operators at workstation $k(k=1, \ldots,|K|)$; it is equal to the workday of operators assigned to the workstation $k: L_{k}=c \cdot T+l_{k}-c$

$\dot{\alpha}_{t}^{+}$Upper limit of dynamic activity factor associated with the $t(t=1, \ldots, T+|K|-1)$ period of the extended workday. This extended workday includes $T$ manufacturing cycles at the first station (total demand) and $|K|-1$ additional cycles that are needed to complete the required work at the last station. Here it is supposed all stations have the same upper limit
} 
$(\forall t: 1 \leq t \leq T+|K|-1)$.

$\dot{\alpha}_{t}^{-} \quad$ Lower limit of dynamic activity factor associated with the $t(t=1, \ldots, T+|K|-1)$ period of the extended workday. Here it is supposed all stations have the same lower limit $(\forall t: 1 \leq t \leq T+|K|-1)$.

$\gamma_{W}$ Cost per work overload unit. It is associated with the production fall that is measured through the work overload.

$\gamma_{b} \quad$ Cost per time unit of a processor.

$\gamma_{U}$ Cost per useless or waste time unit. Here it is supposed $\gamma_{b}=\gamma_{U}$.

Variables

$\rho_{k, t}$ Processing time (at normal activity) required to each homogeneous processor by the $t^{\text {th }}$ product unit at the station $k(k=1, \ldots,|K|)$.

$\hat{v}_{k, t}$ Processing time applied by each processor (at actual activity, $\dot{\alpha}_{k, t}$ ) on the $t^{\text {th }}$ product unit at the station $k(k=1, \ldots,|K|)$.

$\dot{\alpha}_{k, t}$ Dynamic activity factor associated with the $t^{\text {th }}$ operation of the product sequence at the station $k(k=1, \ldots,|K|)$. This factor is calculated from the normal and actual processing times: $\dot{\alpha}_{k, t}=\hat{v}_{k, t} / v_{k, t} \Rightarrow \hat{v}_{k, t}=v_{k, t}\left(\dot{\alpha}_{k, t}\right)$.

$U_{k}$ Useless time by each processor at station $k(k=1, \ldots,|K|)$, measured at normal activity. This time is considered and penalized according the presence time $L_{k}$.

$\Gamma$ Total operating cost: addition of costs by production lost due to overall work overload and the costs of useless time.

And the proposed model, named $M 2_{-} \Gamma$, is as follows:

$$
\begin{array}{ll}
\multicolumn{1}{c}{\operatorname{Min} \Gamma=\Gamma_{W}+\Gamma_{U}=\gamma_{W} \sum_{k=1}^{|K|}\left(b_{k} \sum_{t=1}^{T} w_{k, t}\right)+\gamma_{U} \sum_{k=1}^{|K|} b_{k} U_{k}} \\
\sum_{t=1}^{T} x_{i, t}=d_{i} & (\forall i=1, \ldots,|I|) \\
\sum_{i=1}^{|I|} x_{i, t}=1 & (\forall t=1, \ldots, T) \\
\rho_{k, t}=\sum_{i=1}^{|I|} p_{i, k} x_{i, t} & (\forall k=1, \ldots,|K|) ;(\forall t=1, \ldots, T) \\
v_{k, t}+w_{k, t}=\rho_{k, t} & (\forall k=1, \ldots,|K|) ;(\forall t=1, \ldots, T) \\
v_{k, t}-\dot{\alpha}_{t+k-1}^{+} \cdot \hat{v}_{k, t} \leq 0 & (\forall k=1, \ldots,|K|) ;(\forall t=1, \ldots, T) \\
v_{k, t}-\dot{\alpha}_{t+k-1}^{-} \cdot \hat{v}_{k, t} \geq 0 & (\forall k=1, \ldots,|K|) ;(\forall t=1, \ldots, T) \\
\hat{s}_{k, t} \geq \hat{s}_{k, t-1}+\hat{v}_{k, t-1}-c & (\forall k=1, \ldots,|K|) ;(\forall t=2, \ldots, T) \\
\hat{s}_{k, t} \geq \hat{s}_{k-1, t}+\hat{v}_{k-1, t}-c & (\forall k=2, \ldots,|K|) ;(\forall t=1, \ldots, T) \\
\hat{s}_{k, t}+\hat{v}_{k, t} \leq l_{k} & (\forall k=1, \ldots,|K|) ;(\forall t=1, \ldots, T) \\
U_{k}+\sum_{t=1}^{T} \hat{v}_{k, t}=L_{k} & (\forall k=1, \ldots,|K|)
\end{array}
$$


$U_{k}, \hat{s}_{k, t}, v_{k, t}, \hat{v}_{k, t}, w_{k, t} \geq 0$

$(\forall k=1, \ldots,|K|) ;(\forall t=1, \ldots, T)$

$x_{i, t} \in\{0,1\}$

$(\forall i=1, \ldots,|I|) ;(\forall t=1, \ldots, T)$

$\hat{s}_{1,1}=0$

In the model, the objective function (1.1) represents the minimization of total costs arising to lost production and useless time. Constraints (1.2) - (1.5) force the demand satisfaction, the assignment of products to only one sequence position, the determination of the required processing time and the work overload. The set (1.8) - (1.10) defines the start instants of operations. The new constraints (1.6) and (1.7) are used to reduce the processing times taking into account the maximun and minimum limits for the activity factor. The set (1.11) determines the useless time. Finally, constraints (1.12) - (1.14) establish the initial conditions of variables.

\section{Economic compensation}

Obviously, increasing the activity factor leads to reduced the cost due to the work overload. In addition, the penalization of useless time should be reflected in an increase in the total work completed $(V)$. Because of this, we believe that the excess effort from processors must be compensated.

In line with Bautista, Alfaro-Pozo (2015), we present two metrics to calculate the economic compensation. These are based on establishing an economic value to the exertion unit (e.g. $\left.\gamma_{b}=\gamma_{U}\right)$ and thus, changing effort in monetary units.

1. Economic compensation by extra activity, per station and cycle $\left(g_{k, t}^{1}\right)$ and per station throughout the workday $\left(G_{k}^{1}\right)$.

$$
\begin{array}{ll}
g_{k, t}^{1}=\left\{\begin{array}{l}
\gamma_{b} \cdot b_{k}\left(\dot{\alpha}_{k, t}-1\right) c, \text { if } t=1, \ldots, T-1 \\
\gamma_{b} \cdot b_{k}\left(\dot{\alpha}_{k, t}-1\right) l_{k}, \text { if } t=T
\end{array}\right\} \quad(\forall k=1, \ldots,|K|) ;(\forall t=1, \ldots, T) \\
G_{k}^{1}=\sum_{t=1}^{T} g_{k, t}^{1}=\gamma_{b} \cdot b_{k}\left[\left(\bar{\alpha}_{k}-1\right) c \cdot T+\left(\dot{\alpha}_{k, T}-1\right)\left(l_{k}-c\right)\right] & (\forall k=1, \ldots,|K|)
\end{array}
$$

Where $\bar{\alpha}_{k}$ is the average of dynamic activity factors at station $k \in K$.

2. Economic compensation by recovered processing time $\left(\tilde{v}_{k, t} \equiv v_{k, t}-\hat{v}_{k, t}\right)$, per station and cycle $\left(g_{k, t}^{2}\right)$ and per station throughout the workday $\left(G_{k}^{2}\right)$.

$$
\begin{array}{ll}
g_{k, t}^{2}=\gamma_{b} \cdot b_{k} \cdot \tilde{v}_{k, t}=\left\{\gamma_{b} \cdot b_{k}\left(1-1 / \dot{\alpha}_{k, t}\right) v_{k, t}\right\} & (\forall k=1, \ldots,|K|) ;(\forall t=1, \ldots, T) \\
G_{k}^{2}=\sum_{t=1}^{T} g_{k, t}^{2}=\gamma_{b} \cdot b_{k} \sum_{t=1}^{T} \tilde{v}_{k, t}=\left\{\gamma_{b} \cdot b_{k}\left(V_{k}-\hat{V}_{k}\right)\right\} & (\forall k=1, \ldots,|K|)
\end{array}
$$

Where $\hat{V}_{k}$ is the applied time at workstation $k \in K$. 


\section{Case Study}

From a daily demand plan of the Nissan's Engine Plant in Barcelona (see mix 1 from Bautista, Cano and Alfaro, 2012 - Table 7), we compare the results given by the $M 2_{-} \Gamma$ model againts the results from the reference models, $M 4 \cup 3$ and $M 4 \cup 3 \_\dot{\alpha} I$. The demand plan is made up of a total of $T=270$ production cycles (i.e., product units) in an assembly line with $|K|=21$ workstations; each workstation has one processor $\left(b_{k}=1, \forall k \in K\right)$ that corresponds with two equivalent operators; each processor has a cycle time of $c=175 \mathrm{~s}$ and a time window of $l_{k}=195 \mathrm{~s}$; the cost of one second of work overload, $\gamma_{W}=2.28 € / \mathrm{s}$, is calculated considering the Consolidated Operating Profit of the line (10\% over the profit of one engine, i.e., $400 € /$ engine) and the production cicle $(c=175 s)$; the cost of a useless second, $\gamma_{U}=0.00 \hat{5} € / s$, is determined by the hourly cost in Spain in automitive sector (i.e,. $20 € / h$ ).

In order to evaluate the activation effect we run the models considering the following cases: (1) not consider activation $\left(\alpha^{N}=1.0, \forall k ; \forall t\right)$, i.e., run the $M 4 \cup 3$; (2) consider a linear function that is equivalent to the average value of stepped function (Bautista, Alfaro, Batalla et al., 2015a), with a maximum activation of $3.33 \%$ with respect to the normal activity, $\overline{\alpha^{S}}$, (i.e., $\dot{\alpha}_{k, t}=1.03 \hat{3}, \forall k \in K ; \forall t: 1 \leq t \leq T+|K|-1$ for the $M 4 \cup 3_{-} \dot{\alpha} I$ reference model and $\dot{\alpha}_{t}^{+}=1.03 \hat{3}, \forall t: 1 \leq t \leq T+|K|-1$ for the $M 2_{-} \Gamma$ model).

Obviously, not all work overload and useless time will be due to the production sequence. Indeed, given both a line configuration and a demand plan there will be an unavoidable work overload, $W_{k}^{\circ}\left(\bar{\alpha}_{k}\right)$, that will depend on the station and the activity factor but not on the sequence. On the other hand, there will be also an unavidable useless time, $U_{k}^{\circ}\left(\alpha^{N}\right)$, in regard with the workstation but not with the sequence. These values cannot be minimized. For this reason, after obtaining the solution of the models, the unaviodable work overload and useless time will be deducted, in order to not impute their effect on the production costs; these values are calculated as follows:

$$
\begin{array}{ll}
W_{k}^{\circ}\left(\bar{\alpha}_{k}\right)=b_{k} \cdot \max \left\{0, P_{k}\left(\bar{\alpha}_{k}\right)-L_{k}\right\} & (\forall k=1, \ldots,|K|) \\
U_{k}^{\circ}\left(\alpha^{N}\right)=b_{k} \cdot\left(L_{k}-V_{k}^{\circ}\left(\alpha^{N}\right)\right) & (\forall k=1, \ldots,|K|)
\end{array}
$$

Where $P_{k}\left(\bar{\alpha}_{k}\right)$ is the work required with an average activity, $\bar{\alpha}_{k}$, at the station $k \in K$.

$$
\begin{array}{ll}
P_{k}\left(\bar{\alpha}_{k}\right)=\sum_{i=1}^{|l|} \frac{p_{i, k}}{\bar{\alpha}_{k}} \cdot d_{i} & (\forall k=1, \ldots,|K|) \\
V_{k}^{\circ}\left(\alpha^{N}\right)=P_{k}\left(\alpha^{N}\right)-W_{k}^{\circ}\left(\alpha^{N}\right) & (\forall k=1, \ldots,|K|)
\end{array}
$$


Considering all the set $K$ of workstations, the overall values are the following: $W^{\circ}\left(\bar{\alpha}_{k}\right)=\sum_{k=1}^{|K|} W_{k}^{\circ}\left(\bar{\alpha}_{k}\right) ; \quad V^{\circ}\left(\alpha^{N}\right)=\sum_{k=1}^{|K|} V_{k}^{\circ}\left(\alpha^{N}\right) ; \quad U^{\circ}\left(\alpha^{N}\right)=\sum_{k=1}^{|K|} U_{k}^{\circ}\left(\alpha^{N}\right)$

Therefore, the active work overload and useless time that will be penalysed are:

$\hat{W}=W-W^{\circ}\left(\bar{\alpha}_{k}\right)$

$\hat{U}=U-U^{\circ}\left(\alpha^{N}\right)$

After running the models, $M 4 \cup 3 \_\dot{\alpha} I$ and $M 2_{-} \Gamma$, by the Gurobi v4.6.1 solver, on a Apple Macintosh iMac computer with an Intel Core i72.93 GHz processor and 8 GB of RAM using MAC OS X 10.6.7, with a CPU time limit of 2 hours; and after discounting the unavoidable values of work overload and useless time, we get the following results (Table 1).

Table 1 Values for the unavoidable work overload $\left(W^{\circ}\left(\bar{\alpha}_{k}\right)\right)$ and useless time $\left(U^{\circ}\left(\alpha^{N}\right)\right)($ in seconds), values for the active work overload $(\hat{W})$ and useless time $(\hat{U})$, which are calculated from results given by models (in seconds), daily costs of lost work and unproductive time $\left(\hat{\Gamma}_{W}\right.$ and $\hat{\Gamma}_{U}$ ), total cost of lost work $(\hat{\Gamma})$ and total costs including the possible economic compensations $\left(\hat{\Gamma}+G^{1}\right.$ and $\left.\hat{\Gamma}+G^{2}\right)$.

\begin{tabular}{lccccccccc}
\hline & $W^{\circ}\left(\bar{\alpha}_{k}\right)$ & $U^{\circ}\left(\alpha^{N}\right)$ & $\hat{W}$ & $\hat{U}$ & $\hat{\Gamma}_{W}$ & $\hat{\Gamma}_{U}$ & $\hat{\Gamma}$ & $\hat{\Gamma}+G^{1}$ & $\hat{\Gamma}+G^{2}$ \\
\hline$M 4 \cup 3$ & 50.0 & $185,300.0$ & 256.0 & 92.0 & 585.1 & 0.5 & 585.7 & 585.7 & 585.7 \\
$M 4 \cup 3 \_\dot{\alpha} I\left(\overline{\alpha^{S}}\right)$ & 0.0 & $185,300.0$ & 0.0 & $24,936.8$ & 0.0 & 144.4 & 144.4 & 328.2 & 289.1 \\
$M 2_{-} \Gamma\left(\overline{\alpha^{S}}\right)$ & 0.0 & $185,300.0$ & 0.0 & 389.4 & 0.0 & 2.2 & 2.2 & 4.8 & 4.6 \\
\hline
\end{tabular}

Only the M4U3_d I model reaches the optimal solution with a CPU time of 2 seconds; $M 4 \cup 3$ and $M 2_{-} \Gamma\left(\overline{\alpha^{S}}\right)$ models reach the CPU limit, with a gap to the best bound found by the solver of $83.38 \%$ and $0.14 \%$, respectively. The worst result is given by the model without activation $(M 4 \cup 3)$ because of the work overload value. The reference model, $M 4 \cup 3 \_\dot{\alpha} I\left(\overline{\alpha^{S}}\right)$, despite finishing all work requiered by the demand plan, increases the useless time because it requires an activity factor greater than the normal for all processors throughout the workday. However, the new model eliminates the overload by means of processors' activation when it is needed and avoiding the useless time generation. The latter decreases the compensation costs.

\section{Conclusions}

In this paper we have addressed simultaneously two issues of mixed-model sequences in assembly lines: the work overload elimination and therefore the com- 
pletion of all work required by the demand plan, and the minimization of the useless time of processors, reducing the time during which operators do not add value. For this, a mathematical model has been formulated. This model minimizes the costs incurred for each second of unfinished work and for every second of work that is not used by the processor. Furthermore, in order to increase line productivity without increasing useless time, the model allows free activation of processors, whenever necessary, within the limits established by labor agreements.

Through a case study, we can see how the proposed model means lower costs because it eliminates the workload, reduces useless time and involves less economic compensation by the excess effort of processors.

\section{References}

Alfaro-Pozo R (2015) Modelado y resolución de variantes del problema de secuenciación de modelos mixtos con minimización de la sobrecarga (MMSP-W) con factores de actividad y regularidad en la producción. Doctoral thesis, Universitat Politècnica de Catalunya. Spain, http://hdl.handle.net/10803/363211

Bautista J and Alfaro-Pozo R (2015). Minimización de costes por pérdida de producción y tiempo inerte en el MMSP-WP con activación de los operadores. Technical report, April 2015 http://hdl.handle.net/2117/27690, doi: 10.13140/RG.2.1.3168.9127

Bautista J, Alfaro R, Batalla C and Cano A (2014). Incorporating the Work Pace Concept into the MMSP-W. In Hernández C, López-Paredes A and Pérez-Ríos JM (eds) Managing Complexity: Challenges for Industrial Engineering and Operations Management, 261-268. Springer International Publishing, http://dx.doi.org/10.1007/978-3-319-04705-8_30

Bautista J, Alfaro R and Batalla C (2015a) Modeling and solving the mixed-model sequencing problem to improve productivity. Int J Prod Econ 161: 83-95, doi:10.1016/j.ijpe.2014.11.018

Bautista J, Alfaro-Pozo R and Batalla-García C (2015b) Consideration of human resources in the Mixed-model Sequencing Problem with Work Overload Minimization: Legal provisions and productivity improvement. Expert Sys Appl 42(22): 8896-8910, doi:10.1016/j.eswa.2015.07.044

Bautista J, Cano A and Alfaro R (2012) Models for MMSP-W considering workstation dependencies: A case study of Nissan's Barcelona plant. Eur J Oper Res 223(3): 669-679, doi:10.1016/j.ejor.2012.07.006

Boysen N, Fliedner M and Scholl A (2009) Sequencing mixed-model assembly lines: Survey, classification and model critique, Eur J Oper Res, 192(2): 349-373, doi:10.1016/j.ejor.2007.09.013

Fattahi P and Salehi M (2009) Sequencing the mixed-model assembly line to minimize the total utility and idle costs with variable launching interval. Int J Adv Manuf Tech, 45:987-998, doi: 10.1007/s00170-009-2020-0

Lin D-Y and Chu Y-M (2014) A Lagrangian relaxation approach to the mixed-product assembly line sequencing problem: A case study of a door-lock company in Taiwan. Appl Math Model, 38(17-18): 4493-4511. doi:10.1016/j.apm.2014.02.029

Scholl A, Klein R and Domschke W (1998) Pattern based vocabulary building for effectively sequencing mixed-model assembly lines. J Heuristics 4(4): 359-381, doi: 10.1023/A:1009613925523

Yano C A and Rachamadugu R (1991) Sequencing to minimize work overload in assembly lines with product options. Manage Sci, 37(5): 572-586, http://dx.doi.org/10.1287/mnsc.37.5.572 